\title{
Oscillatory Modes in a Nonlinear Second-Order Differential Equation with Delay
}

\author{
U. an der Heiden, ${ }^{1,6}$ A. Longtin, ${ }^{2,3}$ M. C. Mackey, ${ }^{2-4}$ J. G. Milton, ${ }^{3-5}$ \\ and R. Scholl ${ }^{1}$
}

Received March 23, 1989

\begin{abstract}
Solution properties of the nonlinear second-order delay-differential equation $\ddot{x}(t)=-a x(t)+f[x(t-\tau)]$ are studied where $f$ is a piecewise constant function which mimics negative feedback. We show that the solutions can be obtained by a simple geometrical construction which, in principle, can be implemented using a ruler and a compass. Analytical results guarantee the existence and stability properties of limit cycle solutions. Computer-aided constructions reveal a remarkable richness of different types of dynamical behaviors including a variety of unconventional bifurcation schemes.
\end{abstract}

KEY WORDS: Nonlinear differential equations of second order with deviating argument; oscillations; periodic solutions.

\section{INTRODUCTION}

A particularly interesting class of nonlinear feedback systems is described in the simple form

$$
D^{n} x(t)=f(x(t-\tau))
$$

\footnotetext{
${ }^{1}$ Institut für Mathematik, Universität Witten/Herdecke, Stockumer Strasse 10, D-5810 Witten, F.R.G.

${ }^{2}$ Department of Physics, McGill University, Montréal, Québec, Canada H3A 2 T8.

${ }^{3}$ Center for Nonlinear Dynamics in Physiology and Medicine, McGill University, Montréal, Québec, Canada H3G 1Y6.

${ }^{4}$ Department of Physiology, McGill University, Montréal, Québec, Canada H3G 1Y6.

${ }^{5}$ Department of Neurology, The University of Chicago Hospitals, Chicago, Illinois 60637.

${ }^{6}$ To whom correspondence should be addressed.
} 
Here $t \in \mathbb{R}$ may denote time, $\tau$ is a positive constant representing a delay, $x:[-\tau, \infty] \rightarrow \mathbb{R}$ is some piecewise $n$-times differentiable function, $f: \mathbb{R} \rightarrow \mathbb{R}$ is a piecewise continuous function, and

$$
D^{n} x=\sum_{i=0}^{n} a_{i} \frac{d^{i} x}{d t^{i}}
$$

denotes a differential operator with constants $a_{0}, a_{1}, \ldots, a_{n}$.

The number $n$ gives the order of the equation. For $n=0$ we arrive at the difference equation

$$
x(t)=f(x(t-\tau)),
$$

which, with $f(\xi)=a \xi(1-\xi)$, is well known as the discrete logistic equation or quadratic map. It played a major role in the development of the theory of chaos and period doubling bifurcations (Sarkovskii, 1964; Metropolis et al., 1973; Li and Yorke, 1975; May, 1976; Misiurewicz and Szlenk, 1980).

In the case $n=1$ we obtain

$$
\frac{d x}{d t}(t)=f(x(t-\tau))-a_{0} x(t) .
$$

This difference differential equation has found applications in physics (Furumochi, 1978; Ikeda et al., 1979; Gibbs et al., 1981), population biology (Hutchinson, 1948; Taylor and Sokal, 1976; Gurney et al., 1980; Blythe et al., 1982; May, 1980), medicine (Mackey and Glass, 1977; Mackey, 1978, 1979), neural control (Coleman and Renninger, 1974; Mackey and an der Heiden, 1982; Glass et al., 1988; Longtin and Milton, 1988, 1989a, b; Marcus and Westervelt, 1989), and economics (Mackey, 1989). The time delay, $\tau$, arises because of, for example, finite transmission or production times in feedback loops.

The dynamical behaviors that can be exhibited by Eq. (3) depend on the choice of the feedback function $f$. Negative feedback corresponds to $f$ which is a monotone decreasing function, i.e.,

$$
\xi_{1} \leqslant \xi_{2} \quad \text { implies } f\left(\xi_{1}\right) \geqslant f\left(\xi_{2}\right) .
$$

In this case the existence of periodic solutions has been proved under certain conditions on $\tau, a_{0}$, and the steepness of $f$ (Chow, 1974; Nussbaum, 1974; Hadeler and Tomiuk, 1977; Kaplan and Yorke, 1977). These periodic solutions are of simple type, i.e., they have exactly one maximum per period.

Analytic results (Walther, 1981a) and numerical simulations (Mackey and Glass, 1977; Hadeler, 1980; Saupe, 1982; Longtin and Milton, 1989a) support evidence that these periodic solutions are stable limit cycles. On the other hand, when $f$ is a nonmonotone function, (3) can exhibit very 
complex dynamics including period-doubling bifurcations and chaos, as has been shown both numerically (Mackey and Glass, 1977; Glass and Mackey, 1979) and analytically (Peters, 1980; Walther, 1981b; an der Heiden and Mackey, 1982; an der Heiden and Walther, 1983). For piecewise constant $f$ the existence of invariant measures with ergodic and mixing properties was proved (an der Heiden, 1985).

In contrast to (3), little work has appeared on the study of nonlinear second-order delay-differential equations of the form

$$
\frac{d^{2} x}{d t^{2}}(t)=f(x(t-\tau))-a_{1} \frac{d x}{d t}(t)-a_{0} x(t)
$$

where $a_{0}, a_{1}$ are positive constants [the case $n=2$ in (1)]. Equations of this form provide a better description of many of the systems modeled previously by (3) and, in particular, have been studied in the context of acousto-optical stability (Vallée et al., 1987). More commonly (5) arises in situations describing the delayed feedback control of the movement of mechanical or neuromuscular systems (Maynard Smith, 1968). Physically the left-hand side is interpreted as an acceleration and the right-hand side represents the composition of three forces: (1) a restoring force- $-a_{0} x(t) ;(2)$ a mechanical inertial, or frictional, damping force- $a_{1} d x(t) / d t$; and (3) a delayed restoring force, $f(x(t-\tau))$, dependent on $x(t-\tau)$. Potential applications of (5) arise in the context of the remote control of robotic arms in space (Bejczy and Salisbury, 1983), the sensory feedback control of upper and lower extremity motor prothesis (Phillips, 1988), in the interpretation of experimental studies of the movement of the pupil (Longtin and Milton, 1989b; Milton and Longtin, 1989), and of delayed visual feedback tracking tasks (Reichardt and Poggio, 1976; an der Heiden, 1979a; Glass et al., 1988; Beuter et al., 1989). Delay equations resembling (5), but in which the delay occurs in the damping term, arise in the analysis of the antirolling stabilization of ships (Minorsky, 1962). The existence of nonconstant periodic solutions has been proved for (5) when $a_{1}=a+b$ and $a_{0}=a b$, where $a, b$ are positive constants (an der Heiden, 1979b). However, there is an almost total lack of information concerning the properties of these periodic solutions especially for arbitrary choice of $a_{0}, a_{1}$.

Here we consider the special case of (5) when there is no frictional component, i.e., $a_{1}=0$, and $f$ is a piecewise constant negative feedback function, i.e.,

$$
f(\xi)= \begin{cases}\gamma, & \text { if } \quad \xi \leqslant \beta \\ \delta, & \text { if } \quad \xi>\beta\end{cases}
$$

with constants $\beta, \gamma, \delta, \gamma>\delta$. 
There are two advantages for considering piecewise constant forms for $f$. First, in this case many properties of solutions can be derived analytically without the use of a computer. Second, the dynamics of delayed nonlinear control systems with piecewise constant feedback functions are currently being actively explored in experimental situations, for example, the human pupil light reflex (Longtin and Milton, 1988; Milton and Longtin, 1989; Milton et al., 1988, 1989) and analog electronic circuits (Longtin, Losson, and Cortis, personal communication). These experiments provide unique opportunities to display theoretical predictions and to uncover mathematically unexplained phenomena.

In this paper we show that (5) with $f$ given by (6) possesses a remarkable richness of different types of behavior including a variety of interesting bifurcation schemes.

\section{REDUCING THE NUMBER OF PARAMETERS}

For a nonlinearity $f$ described by (6), solutions to (5) can be constructed geometrically by compass and ruler in the $(x, y)$-plane, where $y=d x / d t$. The reason is that the solution trajectories are given by the piecewise composition of arcs (segments of circles) whose radii and centers are determined by the simple geometric construction developed below.

Before demonstrating this geometric construction, we show how the number of parameters in (5) and (6) may be reduced through a normalization procedure. First, the time scale is transformed linearly by

$$
\bar{t}=\sqrt{a_{0}} t, \quad \bar{x}(\bar{t}):=x(t)
$$

With $a_{1}=0$, Eq. (5) then becomes

$$
\frac{d^{2} \bar{x}}{d \bar{t}^{2}}(\bar{t})=a_{0}^{-1} f\left(\bar{x}\left(\bar{t}-\sqrt{a_{0}} \cdot \tau\right)\right)-\bar{x}(\bar{t})
$$

Equation (8) shows that, without any loss of generality, we can assume $a_{0}=1$ in Eq. (5). An additional normalization can be obtained by the linear transformation

$$
\bar{x}=(\gamma-\delta) \tilde{x}+(\gamma+\delta) / 2
$$

With the exception of the trivial case of $\gamma=\delta$, corresponding to the linear homogeneous equation for the harmonic oscillator, Eq. (8) transforms into

$$
\frac{d^{2} \tilde{x}(t)}{d t^{2}}=-\tilde{x}(t)+\left\{\begin{array}{ccc}
1 / 2 & \text { if } & \tilde{x}(t-\tau) \leqslant\left(\beta-\frac{\gamma+\delta}{2}\right) /(\gamma-\delta) \\
-1 / 2 & \text { if } & \tilde{x}(t-\tau)>\left(\beta-\frac{\gamma+\delta}{2}\right) /(\gamma-\delta)
\end{array}\right.
$$


Thus with $a_{1}=0$, the solutions of (5) and (6) are parametrically dependent on only the time delay $\tau$ and a threshold $\Theta$ defined by

$$
\Theta:=\left(\beta-\frac{\gamma+\delta}{2}\right) /(\gamma-\delta)
$$

With these transformations we arrive at the final form of the differential equation under investigation:

$$
\frac{d^{2} x}{d t^{2}}(t)=f(x(t-\tau))-x(t)
$$

where

$$
f(\xi)=\left\{\begin{array}{rll}
1 / 2 & \text { if } \quad \xi \leqslant \Theta \\
-1 / 2 & \text { if } & \xi>\Theta
\end{array}\right.
$$

with constants $\tau>0, \Theta \in \mathbb{R}$.

\section{EXISTENCE AND BASIC PROPERTIES OF SOLUTIONS}

A geometric construction by means of compass and ruler of the solutions of (12), (13) can be achieved if Eq. (12) is replaced by the equivalent system of equations

$$
\begin{aligned}
& \frac{d x}{d t}(t)=y(t) \\
& \frac{d y}{d t}(t)=f(x(t-\tau))-x(t) .
\end{aligned}
$$

An initial condition is given by some pair $\left(x_{0}, y_{0}\right)$, where $x_{0}$ is a differentiable function $x_{0}:[-\tau, 0] \rightarrow \mathbb{R}$ and $y_{0} \in \mathbb{R}$. Moreover, it is assumed throughout that $x_{0}(t)=\Theta$ for at most finitely many $t \in[-\tau, 0]$.

Associated with each such initial condition there is a unique solution $(x, y)$ satisfying the conditions a-d:

(a) $x:[-\tau, \infty) \rightarrow \mathbb{R}$ is differentiable;

(b) $y:[0, \infty) \rightarrow \mathbb{R}$ is continuous for all $t \in[0, \infty)$ and differentiable for all $t$ such that $x(t-\tau) \neq \Theta$;

(c) the pair of functions $(x, y)$ satisfies the equations (14) for all $t>0$ obeying $x(t-\tau) \neq \Theta$, with $f$ given by (13);

(d) $x(t)=x_{0}(t)$ for all $t \in[-\tau, 0]$, $y(0)=y_{0}$. 
This existence theorem is established by the following observations, which are basic for this paper. Since $f$ has only the value $+1 / 2$ and $-1 / 2$, any solution $(x, y)$ of (14) must satisfy the following condition: if $-\tau \leqslant t_{1}<t_{2}$ and $x(t) \leqslant \Theta$ for all $t \in\left(t_{1}, t_{2}\right)$ or $x(t)>\Theta$ for all $t \in\left(t_{1}, t_{2}\right)$, then there are numbers $A, \varphi \in \mathbb{R}$ such that

$$
\left.\begin{array}{l}
x(t)= \pm 1 / 2+A \sin (t+\varphi) \\
y(t)=A \cos (t+\varphi)
\end{array}\right\} \quad \text { for all } \quad t \in\left[t_{1}+\tau, t_{2}+\tau\right]
$$

the positive (negative) sign holds if $x(t) \leqslant \Theta(x(t)>\Theta)$ for all $t \in\left(t_{1}, t_{2}\right)$.

Thus, any solution $(x, y)$ is necessarily a piecewise and continuous composition of arcs (or sections) of circles described by (15). The arcs are parametrized by $t$ and can be considered to be located in the plane $\mathbb{R}^{2}$. (The normalization and parameter reduction in the previous section is responsible for this simple solution behavior consisting of composition of arcs. Had this procedure not been carried out, the solutions would have consisted of sections of ellipses.) If $x(t) \leqslant \Theta$ for all $t \in\left(t_{1}, t_{2}\right)$, then the center of the arc parametrized by $t \in\left[t_{1}+\tau, t_{2}+\tau\right]$ is located at $(1 / 2,0)$. Respectively, if $x(t-\tau)>\Theta$, then the center of the arc is located at $(-1 / 2,0)$. A switching from one of these centers to the other occurs at $t_{1}+\tau$ iff $x(t)-\Theta$ changes its sign from nonpositive to positive at time $t=t_{1}$. The conditions on the initial function $x_{0}$ guarantee that switching occurs only at discrete points in time. Note that because of this switching the component $y$ is not differentiable at such a time $t_{1}+\tau$.

The amplitude (radius) $A$ and the phase $\varphi$ of the arc (15) are uniquely determined by the following condition. Assume the solution is known up to the time $t_{1}+\tau$, in particular, $x\left(t_{1}+\tau\right)$ and $y\left(t_{1}+\tau\right)$ are given. Since solutions of Eq. (14) are required to be continuous, the amplitude $A$ and the phase $\varphi$, valid for the interval $\left[t_{1}+\tau, t_{2}+\tau\right]$, must obey

$$
x\left(t_{1}+\tau\right)= \pm 1 / 2+A \sin \left(t_{1}+\tau+\varphi\right), \quad y\left(t_{1}+\tau\right)=A \cos \left(t_{1}+\tau+\varphi\right)
$$

[the sign in front of $1 / 2$ is determined as in (15)]. The system (16) of nonlinear equations uniquely determines the unknown $A$ and $\varphi$.

By induction, sequences of times $\left(\tau_{i}\right)$, amplitudes $\left(A_{i}\right)$, and phases $\left(\varphi_{i}\right)$, $i=1,2, \ldots$, are uniquely determined such that

$$
\left.\begin{array}{rl}
0 & =\tau_{1}, \tau_{i}<\tau_{i+1}, \quad \lim _{i \rightarrow \infty} \tau_{i}=\infty \\
x(t) & = \pm 1 / 2(-1)^{i}+A_{i} \sin \left(t+\varphi_{i}\right) \\
y(t) & =A_{i} \cos \left(t+\varphi_{i}\right)
\end{array}\right\} \quad \text { for all } t \in\left[\tau_{i}, \tau_{i+1}\right]
$$

where the plus sign holds if $x(-\tau)=x_{0}(-\tau) \leqslant \Theta$ and the minus sign if $x_{0}(-\tau)>\Theta$. [For the exceptional case $x_{0}(-\tau)=\Theta$ the sign depends 
correspondingly on $x_{0}(-\tau+\varepsilon)$ with sufficiently small $\varepsilon>0$.] Obviously the functions $x, y$ defined in this way satisfy the conditions a-d above and henceforth represent the unique solution.

\section{GEOMETRIC CONSTRUCTION OF SOLUTIONS}

Making use of the observations in the previous section, solutions can be constructed by compass and ruler as follows. We illustrate the method in Fig. 1a, where $\tau=\frac{3}{4} \pi$ and $\Theta=1 / 2$.

Let the initial condition be $x_{0}(t)=1 / 4$ for all $t \in[-\tau, 0]$ and $y(0)=0$. Since $x(t)<\Theta$ for $t \in[-\tau, 0]$ it follows from (15) with $t_{1}=-\tau, t_{2}=0$ that for $t \in[0, \tau]$ the solution $x(t), y(t)$ describes an arc in the $(x, y)$-plane with center at $(1 / 2,0)$. The arc is parametrized by $t$, thus it is extended across an angle of length $\tau=3 \pi / 4$. The arc is uniquely determined by its angular length $(3 \pi / 4)$, its center $(1 / 2,0)$, and its initial point $\left(x_{0}, y_{0}\right)=(1 / 4,0)$. These values allow a simple construction by compass and ruler.

Note that $x(t)<\Theta$ for all $t \in(0, \pi / 2)$ and $x(\pi / 2)=\Theta$. Thus, according to (15) with $t_{1}=0$ and $t_{2}=\pi / 2$, the solution is continued to the time interval $[\tau, \tau+\pi / 2]=[3 \pi / 4,5 \pi / 4]$ by a second arc. The center of this arc is again $(1 / 2,0)$ because $x(t)<\Theta$ for $t \in[0, \pi / 2]$. Its angular length is just the length of this time interval, namely, $\pi / 2$, and its initial point coincides with the end point of the previous arc. Thus it is uniquely determined and constructable. In Fig. 1a the end point of this arc is labeled $\tau_{2}$ according to the meaning of $\tau_{i}$ introduced above.

The next arc is obtained as follows. Since $x(t)>\theta$ during the time interval $(\pi / 2,5 \pi / 4)$ its angular length is $5 \pi / 4-\pi / 2=3 \pi / 4$ and its center is $(-1 / 2,0)$. Its starting point is, as always, the end point of the previous arc (note that in this way the amplitude of the arc is specified). This arc crosses the threshold line $\Theta=1 / 2$ at a point indicated in Fig. 1a by $\tau_{3}-\tau$. This notation highlights the fact that the arc around $(-1 / 2,0)$ must be prolonged such that its end point (indicated by $\tau_{3}$ ) has an angular distance $\tau$ from that threshold crossing point. The arc around $(1 / 2,0)$ from time $\tau_{3}$ to time $\tau_{4}$ is drawn in a completely analogous fashion.

Let us now describe the construction method quite generally for all values of the parameters $\tau$ and $\Theta$. The method crucially depends on the principle, following from (15), that time parametrizes the angular lengths of the arcs out of which each solution is composed. In other words, the angular velocity is always equal to one, independent of the centers and amplitudes of the arcs. Note that the evolution of the arcs is always in the positive (clockwise) direction, as follows from (15). Assume the solution is already constructed up to a time $t=\bar{t}$ such that at time $\bar{t}-\tau$ a crossing of the threshold $\Theta$ took place, i.e., $x(\bar{t}-\tau)=\Theta$. Assume additionally that 
$d x(\bar{t}-\tau) / d t>0$ (the situation with the opposite inequality is analogous). Then there is a maximal time $t^{*}$ satisfying $\bar{t}-\tau<t^{*} \leqslant \bar{t}$ and $x(t)>\Theta$ for all $t \in\left(\bar{t}-\tau, t^{*}\right)$. The relation (15) implies again that for all $t \in\left[\bar{t}, t^{*}+\tau\right)$ the solution $(x(t), y(t))$ is an arc with center at $(-1 / 2,0)$, starting at $(x(\bar{t})$, $y(\bar{t}))$ and having angular length $t^{*}+\tau-\bar{t}$.

This arc can be drawn by compass and ruler, because its angular length $t^{*}+\tau-\bar{t}$ can be determined by compass and ruler from the trajectory in the $(x, y)$-plane extending from $(x(\bar{t}-\tau), y(\bar{t}-\tau))$ to $\left(x\left(t^{*}\right)\right.$, $\left.y\left(t^{*}\right)\right)$. After this arc has been drawn the next step depends on whether $x\left(t^{*}\right)=\Theta$ or $x\left(t^{*}\right)>\Theta$. In the first case define a new $\bar{t}$ by $\bar{t}=t^{*}+\tau$ and proceed as before with the exception that now $d x(\bar{t}-\tau) / d t=d x\left(t^{*}\right) / d t<0$ and the center of the next arc is $(1 / 2,0)$.

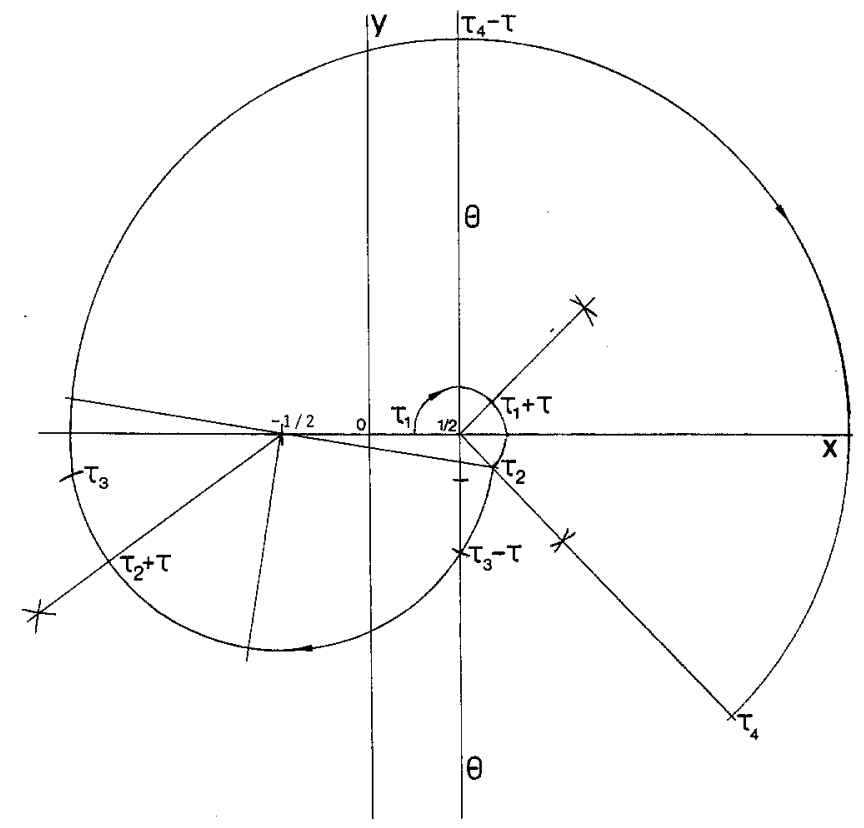

(a)

Fig. 1. (a) Construction by compass and ruler of a trajectory of the system described by Eqs. (13) and (14). Trajectories are piecewise composed of ares with center either at $(-1 / 2,0)$ or at $(1 / 2,0)$. For details of the construction see the text. Parameters $\Theta=1 / 2, \tau=3 \pi / 4$. Initial condition $x_{0} \equiv 1 / 4, y_{0}=0$. Symbols $\tau_{1}$ indicate times where center of arcs changes. Theorem 1 shows that the spiral becomes infinitely large as $t \rightarrow \infty$. (b) As in (a), but now $\Theta=0, \tau=5 \pi / 4$. Theorem 2 shows that the trajectory converges towards a periodic orbit consisting of two arcs each having angular length $\tau$. Thus, the period is $2 \tau$. (c) As in (a), but now $\Theta=1 / 2, \tau=3 \pi / 2$. The figure shows a single periodic orbit which is immediately entered from the initial condition $\left(x_{0} \equiv 1 / 4, y_{0}=0\right)$. One orbit consists of three circles, has a period $6 \pi=4 \tau$, and is traced by following the numbered half circles sequentially. 


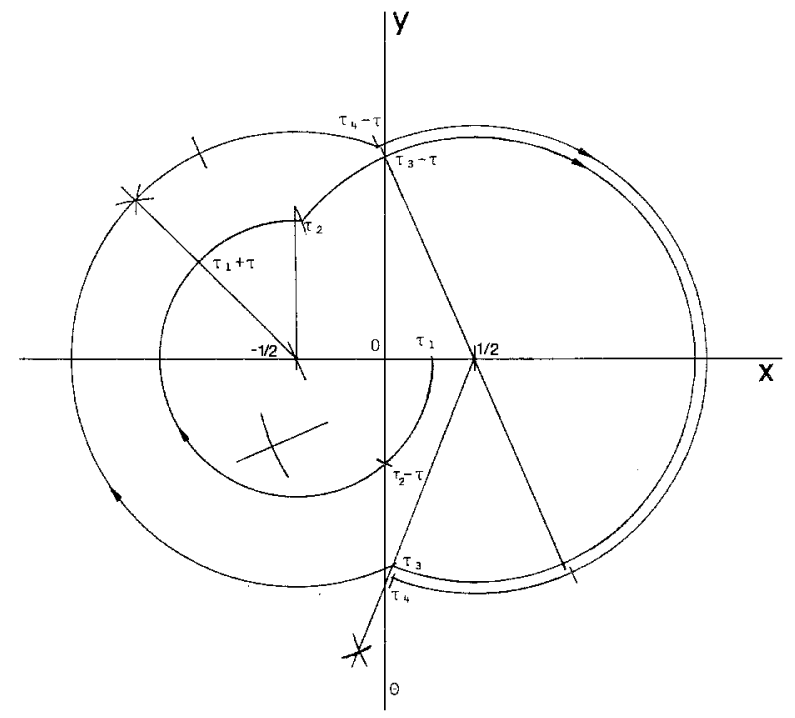

(b)

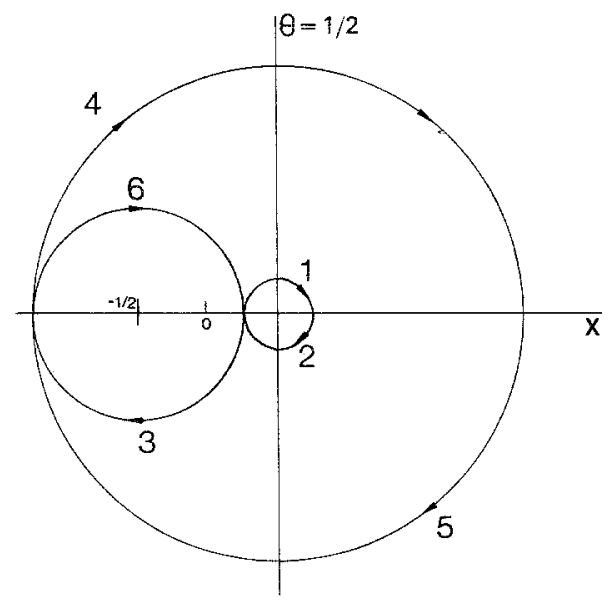

(c)

Fig. 1. Continued.

In the second case no change of the arc center takes place. Instead, the arc just drawn is continued until the first time $t>t^{*}+\tau>t$ where it happens that $x(t-\tau)=\Theta$. This can be done by compass and ruler since time is represented by angles and under the assumption that $\tau$ is given by some width of the compass. Defining hereafter a new value $t=t$ we arrive 
at the first case, and the solution can again be continued as described there. Thus we always end up in a situation where $x(t-\tau)=\Theta$ and we have described how to proceed in such a situation. Therefore the solution can be continued for arbitrary (finite) times. [Note that if in the beginning there is no time $t$ with $x(t-\tau)=\Theta$, then depending on $x(-\tau) \leqslant \Theta$ or $x(-\tau)>\Theta$, one simply draws an arc around $(1 / 2,0)$ or $(-1 / 2,0)$ respectively, starting at $(x(0), y(0))$ until the condition $x(t-\tau)=\Theta$ is eventually satisfied.]

This completes the description of the general construction method. The reader may use the examples in Figs. 1a $\mathrm{c}$ to study these construction rules. As far as shown this solution is a spiral moving outward. It is proved in the following section that this spiral extends to infinity and the solution is unbounded.

Figure $1 \mathrm{~b}$ shows the construction of a bounded solution occurring for parameters $\tau=\frac{5}{4} \pi, \Theta=0$. In the next section it is proved that this trajectory converges toward a limit cycle which is asymptotically orbitally stable with respect to a certain class of initial conditions.

A third example is shown in Fig. 1c. Here $\tau=3 \pi / 2$ and $\Theta=1 / 2$. A periodic solution is obtained whose orbit is composed of two small and one large circle. Thus, the (smallest) period is $6 \pi$. This periodic orbit is immediately entered after starting with a constant initial condition [here $x_{0}(t)=1 / 4$ for $\left.t \in[-\tau, 0], y_{0}=y(0)=0\right]$. Similarly the reader will easily realize that to each constant initial condition $x_{0} \in[-1 / 2,1 / 2], y_{0}=0$, there belongs a separate periodic orbit. Each of these orbits consists of three circles with radii $1 / 2-x_{0}, x_{0}+1 / 2$, and $3 / 2+x_{0}$. Thus, none of these periodic orbits is asymptotically stable, and we observe a rather strange center structure.

For other values of the parameters $\tau$ and $\Theta$ the behavior of the solutions may be very complicated, and construction by compass and ruler may become very tedious. In such situations it is much more practical to use a computer for the construction. It is important to emphasize that we did not use any of the classical routines for numerical integration of differential equations (as, e.g., the Runge-Kutta method) but rather used the representation (15) of solutions in the following way.

Assume a solution is already known in the time interval $[t-\tau, t]$. Let $\left\{t_{1}, t_{2}, \ldots, t_{k}\right\}$ be the set of times for which $x\left(t_{i}\right)=\Theta$ and where $x(t-\tau)$ changes sign. Then in the intervals $\left[t, t_{1}+\tau\right],\left[t_{1}+\tau, t_{2}+\tau\right], \ldots,\left[t_{k}+\tau, t+\tau\right]$ the solution can be calculated by the expression (15), where the sign in front of $1 / 2$ alternates from interval to interval. $A$ and $\varphi$, which generally vary from interval to interval, are uniquely determined by the condition that the solution has to be continuous at each $t_{i}$ [compare (16)]. In this way the solution is precisely extended to $[t, t+\tau]$ (and iteratively to arbitrary long times). Determination of the intersection times $t_{i}$ is accom- 
plished by using the inverse trigonometric functions and (15). These computer-aided constructions are used in a later section to develop further insight into the nature of the solution behavior.

\section{ANALYTIC DISCUSSION OF SOLUTIONS}

The system (12), (13) is one of the very few nonlinear differential equations where solutions (or parts of them) can be calculated analytically and proofs of the qualitative behavior of solutions can be given. In this section we demonstrate some of the simpler properties.

Throughout this section we restrict our attention to the symmetric situation where $\Theta=0$. Thus, the only free parameter is the delay $\tau$.

For $\Theta=0$ the nonlinearity $f$ is an odd function. It follows easily from (13) and (14) that this symmetry condition of the differential equation implies the following symmetry property of the solutions.

Lemma 1. Let $\Theta=0$. If $(x, y)$ is the solution of (13) and (14) corresponding to the initial condition $\left(x_{0}, y_{0}\right)$, then $(-x,-y)$ is the solution of (13) and (14) corresponding to the initial condition $\left(-x_{0},-y_{0}\right)$.

The following theorem essentially says that if the delay satisfies $0<\tau \leqslant \pi$, then for a large class of initial conditions solutions grow toward infinity in the form of an expanding spiral. The case $\tau=0$, where bounded solutions exist, is treated in Corollary 1.

Theorem 1. Let $\Theta=0$ and $0<\tau \leqslant \pi$. Let the initial condition $\left(x_{0}, y_{0}\right)$ satisfy $x_{0}(0)=0, x_{0}(t)>\Theta$ for $t \in(-\tau, 0), y_{0} \leqslant 0$. Then the corresponding solution $(x, y)$ of the system (13) and (14) has the following properties:

(i) the solution oscillates in a way characterized by a sequence $\left(t_{i}\right)$ with $t_{i} \rightarrow \infty$ as $i \rightarrow \infty, x\left(t_{2 i}\right)=0$, and $x\left(t_{2 i+1}\right) \neq 0$ for all $i \in \mathbb{N}$.

(ii) the solution becomes infinitely large, i.e., $\|(x(t), y(t) \| \rightarrow \infty$ as $t \rightarrow \infty$.

Proof. Let $x_{0}$ be some initial condition described in the theorem. Let $P_{0}=\left(0, y_{0}\right)$. Then, because of $(15)$, for $t \in[0, \tau]$ the corresponding solution $(x(t), y(t))$ describes an oriented arc in the $(x, y)$-plane with center at $(-1 / 2,0)$, starting at point $P_{0}$, and having angular length $\tau$ (for illustration see Fig. 2a). Let $P_{1}$ be the end point of this arc. Let $r_{0}$ be the radius of this arc, i.e., $r_{0}$ is the distance between the points $(-1 / 2,0)$ and $P_{0}$. Note $r_{0} \geqslant 1 / 2$.

Since $x(t)<\Theta=0$ for $t \in(0, \tau)$ it follows from (15) that for $t \geqslant \tau$ the solution $(x(t), y(t))$ is continued by an oriented arc around $(1 / 2,0)$ starting at point $P_{1}$ and extending at least until the first point $P_{2}$, where this arc 


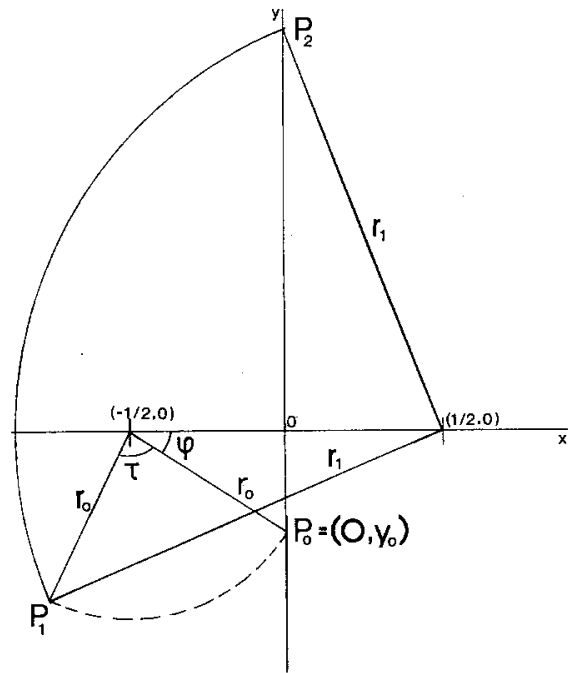

(a)

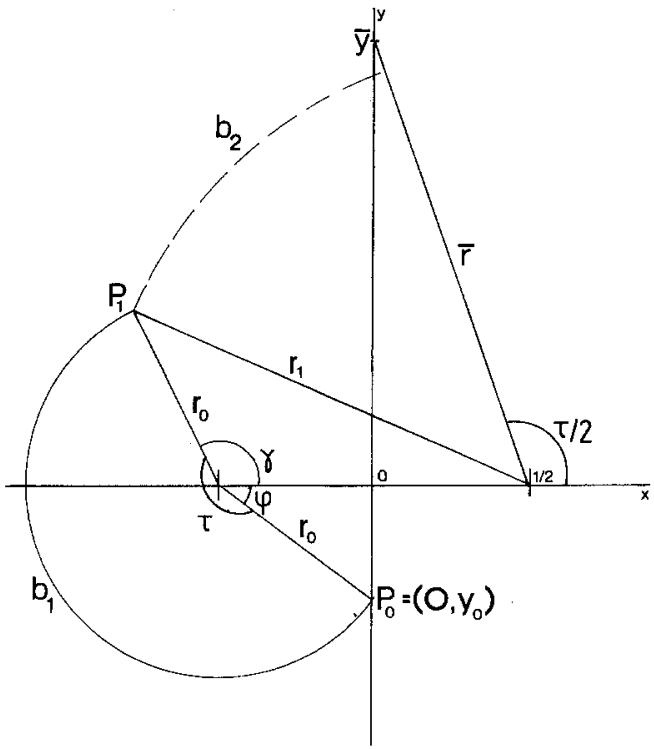

(b)

Fig. 2. (a) Illustration for the proof of Theorem 1. (b) Illustration for the proof of Theorem 3. 
meets the $y$-axis (for illustration see Fig. 2a). Define $P_{2}$ as the end point of this arc and let $t^{*}$ denote the time where $\left(x\left(t^{*}\right), y\left(t^{*}\right)\right)=P_{2}$. Let $r_{1}$ be the radius of this arc. Thus, for $t \in\left[0, t^{*}\right]$ the solution is composed of two arcs, one with radius $r_{0}$ and the other with radius $r_{1}$. We now compute $r_{1}$ as a function $\left(g_{\tau}\right)$ of $r_{0}$. Consider the triangle with corners $(-1 / 2,0), P_{1}$, and $(1 / 2,0)$. Its sides have lengths $r_{0}, 1$, and $r_{1}$. The angle at the point $(-1 / 2,0)$ is equal to $\tau+\varphi$, where

$$
\cos \varphi=\frac{1}{2 r_{0}}
$$

Applying the cosine theorem we obtain

$$
r_{1}^{2}=1+r_{0}^{2}-2 r_{0} \cos (\tau+\varphi)
$$

The addition theorem gives

$$
\cos (\tau+\varphi)=\cos \tau \cos \varphi-\sin \tau \sin \varphi=\frac{\cos \tau}{2 r_{0}}-\frac{\sqrt{r_{0}^{2}-1 / 4}}{r_{0}} \sin \tau
$$

Hence,

$$
r_{1}^{2}=1+r_{0}^{2}-\cos \tau+2 \sqrt{r_{0}^{2}-1 / 4} \sin \tau=g_{\tau}^{2}\left(r_{0}\right)
$$

and

$$
r_{1}=r_{0} \quad \text { if } \quad \tau=0 ; \quad r_{1}>r_{0} \quad \text { if } \quad 0<\tau \leqslant \pi
$$

Equation (20) describes the dependence $g_{\tau}$ of $r_{1}$ on $r_{0}$ for fixed values of $\tau$. For the further development of the solution beyond the time $t^{*}$ we take advantage of Lemma 1 . Note that for $t \in\left[t^{*}-\tau, t^{*}\right]$ the solution obeys $x(t)<\Theta=0$; moreover, $x\left(t^{*}\right)=0$ and $y\left(t^{*}\right) \geqslant 0$. These properties are just opposite in sign to those of the initial condition formulated in the theorem. Therefore with Lemma 1 the solution proceeds from the new initial time $t^{*}$ (replacing the initial time $t=0$ ) just as from $t=0$, however, with opposite sign: First, there is an arc around $(1 / 2,0)$ starting from $P_{2}$ (replacing $P_{0}$ ) with radius $r_{1}$ (replacing $r_{0}$ ) and of angular length $\tau$, ending in a point $P_{3}$ (replacing $P_{1}$ ). Afterward it follows an arc around $(-1 / 2,0)$ starting at $P_{3}$ and ending on the $y$-axis in some point $P_{4}$. Of course the radius $r_{2}$ of this arc satisfies $r_{2}=g_{\tau}\left(r_{1}\right)$, where $g_{\tau}$ is defined by (20); moreover,

$$
r_{2}=r_{1} \quad \text { if } \quad \tau=0, \quad \text { and } \quad r_{2}>r_{1} \quad \text { if } \quad 0<\tau \leqslant \pi
$$

By induction, the solution is composed of a sequence $\left(a_{i}\right), i \in \mathbb{N}$, of $\operatorname{arcs}$ with radii $r_{i}$ satisfying

$$
r_{i+1}=g_{\tau}\left(r_{i}\right)
$$


where $g_{\tau}:[1 / 2, \infty) \rightarrow[1 / 2, \infty)$ is defined by

$$
g_{\tau}(r)=\sqrt{1+r^{2}-\cos \tau+2 \sqrt{r^{2}-1 / 4} \sin \tau}
$$

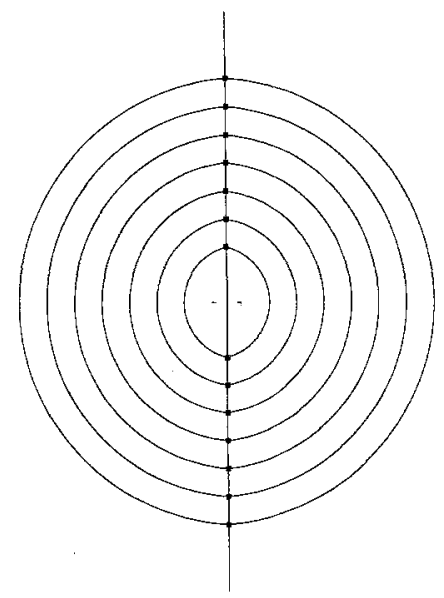

(a)

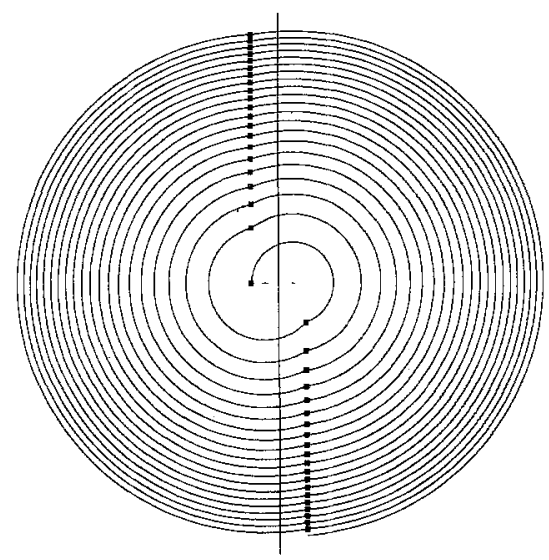

(b)

Fig. 3. (a) Computer-aided construction of trajectories of the system described by Eqs. (13) and (14). Parameters $\Theta=0, \tau=0$. The figure shows seven periodic orbits belonging to initial conditions $\left(x_{0}, y_{0}\right)$ with $x_{0} \equiv 0$ always and $y_{0}=2,3, \ldots, 8$. Existence of infinitely many periodic orbits is proved by Corollary 1 . Throughout all the following figures, black squares indicate switch of center. The centers $(-1 / 2,0)$ and $(1 / 2,0)$ are indicated by crosses. Vertical bar indicates the threshold $\Theta$. Time orientation of trajectories is always clockwise. (b) As in (a), but now $\Theta=0, \tau=\pi$. Initial condition $x_{0} \equiv 1, y_{0}=0$. Trajectory of "expanding spiral" type. Theorem 1 proves that the special is infinitely large. 
The arcs $a_{i}$ with odd $i$ have center at $(-1 / 2,0)$; the arcs $a_{i}$ with even $i$ have center at $(+1 / 2,0)$.

Since in case of $\tau>0$ we have $g_{\tau}(r)>r$ for all $r \geqslant 1 / 2$, it follows that $r_{i} \rightarrow \infty$ as $i \rightarrow \infty$.

An example of one of the solutions covered by this theorem is shown in Fig. 3b.

The proof of the theorem has also shown [note that $g(r)=r$ for $\tau=0$ ] the following.

Corollary 1. Let $\Theta=0$ and $\tau=0$. Then the system (13) and (14) has infinitely many different periodic solutions. Each periodic orbit consists of exactly two arcs, one with center at $(-1 / 2,0)$, the other at $(1 / 2,0)$.

An illustration of these periods is given in Fig. 3a.

The following theorem shows that if the delay satisfies $\pi<\tau \leqslant 2 \pi$, then bounded solutions which are periodic exist. An illustration of corresponding periodic orbits is shown for several values of $\tau$ in Figs. 5a and b.

Theorem 2. Let $\Theta=0$ and $\pi<\tau \leqslant 2 \pi$. Then the system (13) and (14) has a nonconstant periodic solution. Its period is $2 \tau$.

Proof. We first give an explicit description of the periodic orbit. Afterward it will be easily seen that this orbit corresponds to a periodic solution of (13) and (14). The orbit is composed of two arcs, $a_{1}$ and $a_{2}$, in the $(x, y)$-plane, each having angular length $\tau$. The arc $a_{1}$ is situated entirely to the left of the $y$-axis, its center is at $(-1 / 2,0)$, and its starting point is $(0, \bar{y})$, where

$$
\bar{y}=\frac{1}{2} \tan (\pi-\tau / 2)
$$

Its end point is $(0, \bar{y})$. The $\operatorname{arc} a_{2}$ has the center $(1 / 2,0)$ and is entirely situated to the right of the $y$-axis, its starting point is $(0, \bar{y})$, and its end point is $(0,-\bar{y})$. This completes the description of the periodic orbit. In order to realize that this orbit is the trajectory of a periodic solution, it is sufficient to observe that the angular length of each of the two arcs is just equal to the delay $\tau$. Thus, if one starts with one of the two arcs as an initial condition in the time interval $[-\tau, 0]$, then according to (15) the other arc is obtained as a solution trajectory in the time interval $[0, \tau]$. By induction it follows that the described orbit is the trajectory of a periodic solution.

Q.E.D.

Computer graphic investigations suggest that the periodic orbit of 
Theorem 2 is a stable limit cycle for all $\tau$ satisfying $\pi<\tau<\tau_{\mathrm{c}}$, where $\tau_{\mathrm{c}}$ is some critical value of the delay,

$$
\pi<\tau_{\mathrm{c}}<2 \pi
$$

and that it is an unstable limit cycle if $\tau_{\mathrm{c}}<\tau \leqslant 2 \pi$.

It is not possible to prove the stability (attractiveness) of the limit cycle (for $\pi<\tau<\tau_{c}$ ) with respect to arbitrary solutions. However, the following theorem shows that at least for a certain class of solutions, it can be demonstrated analytically that their trajectories approach the periodic orbit described in the proof of Theorem 2 .

Theorem 3. Assume that the following conditions hold:

$$
\begin{aligned}
& \text { (i) } \Theta=0 ; \\
& \text { (ii) } \pi<\tau<2 \pi \text {, cos } \tau<-3 / 4 \\
& \text { (iii) } x_{0}(t)>\Theta \text { for all } t \in[-\tau, 0], x_{0}(0)=\Theta \text {; and } \\
& \text { (iv) }-\bar{y}<y_{0} \leqslant 0 \text { [where } \bar{y} \text { is defined by (25)]. }
\end{aligned}
$$

Then the trajectory corresponding to the initial condition $\left(x_{0}, y_{0}\right)$ converges toward the periodic orbit described in Theorem 2.

Proof. Similar to the proof of Theorem 1, for $t \in[0, \tau]$ the solution (corresponding to such an $\left.\left(x_{0}, y_{0}\right)\right)$ describes an $\operatorname{arc} b_{1}$ around $(-1 / 2,0)$ starting at $P_{0}=\left(0, y_{0}\right)$ and having angular length $\tau$ (see Fig. $2 \mathrm{~b}$ ). Denote the radius of this arc by $r_{0}$ and its end point by $P_{1}$. This arc is followed by an $\operatorname{arc} b_{2}$ around $(1 / 2,0)$ starting at $P_{1}$. The radius $r_{1}$ of this arc is given by the distance between $P_{1}$ and $(1 / 2,0)$. Since $y_{0} \geqslant-\bar{y}$ the arc $b_{1}$ is entirely situated to the left of the $y$-axis. Therefore and because of (15) the arc $b_{2}$ intersects the $y$-axis at some point $P_{2}$.

We now determine $r_{1}$ as a function $g$ of $r_{0}$ :

$$
g:[1 / 2, \bar{r}] \rightarrow[1 / 2, \bar{r}], \quad r_{1}=g\left(r_{0}\right)
$$

where $\bar{r}$ denotes the distance between $(0, \bar{y})$ and $(1 / 2,0), \quad \bar{r}=$ $1 /[2 \cos (\pi-\tau / 2)]$. Consider the triangle defined by the points $(1 / 2,0), P_{1}$, $(-1 / 2,0)$. The angle $\gamma$ at $(-1 / 2,0)$ satisfies

$$
\gamma=2 \pi-\tau-\varphi, \quad \text { where } \quad \cos \varphi=\frac{1}{2 r_{0}}
$$

(compare with Fig. 2b). The cosine theorem gives

$$
r_{1}^{2}=1+r_{0}^{2}-2 r_{0} \cos \gamma
$$


while from the addition theorem we have

$$
\begin{aligned}
\cos \gamma & =\cos (2 \pi-\tau) \cos \varphi+\sin (2 \pi-\tau) \sin \varphi \\
& =\frac{1}{2 r_{0}} \cos (2 \pi-\tau)+r_{0}^{-1} \sqrt{r_{0}^{2}-1 / 4} \sin (2 \pi-\tau)
\end{aligned}
$$

The function $g$ is obtained from (29) and (30):

$$
r_{1}=g\left(r_{0}\right)=\sqrt{1+r_{0}^{2}-\cos (2 \pi-\tau)-2 \sqrt{r_{0}^{2}-1 / 4} \sin (2 \pi-\tau)}
$$

It is easily seen that in the domain $r \geqslant 1 / 2$ there is a unique fixed point of $g$ and that this fixed point equals $\bar{r}$ :

$$
g(\bar{r})=\bar{r}
$$

Because of the assumption (ii) on $\tau$ it follows that

$$
1 / 2<g(1 / 2)<\bar{r}
$$

The uniqueness of the fixed point of $g$ and (33) imply

$$
g(r)>r \quad \text { for all } r \in[1 / 2, \bar{r})
$$

To establish (27) we still have to show that $g(r) \leqslant \bar{r}$ for all $r \in[1 / 2, \bar{r})$. This inequality follows easily from the fact that the derivative $g^{\prime}(r)$ is negative for $r<\sin ^{2}(2 \pi-\tau)+1 / 4$ and positive for $r>\sin ^{2}(2 \pi-\tau)+1 / 4$. iteration

The relations (27), (32), and (34) imply that for any $r_{0} \in[1 / 2, \vec{r})$ the

$$
r_{i+1}=g\left(r_{i}\right), \quad i \in \mathbb{N} \cup\{0\}
$$

satisfies

$$
r_{i} \in[1 / 2, \bar{r}], \quad r_{i} \rightarrow \bar{r} \quad \text { as } \quad i \rightarrow \infty
$$

The proof of the theorem for situations where $y_{0} \geqslant-\bar{y}$ is completed by the observation that the two arcs $b_{1}$ and $b_{2}$ with end points $P_{0}, P_{1}$ and $P_{1}, P_{2}$, respectively, and radii $r_{0}, r_{1}=g\left(r_{0}\right)$ are situated to the left of the $y$-axis. Because of the symmetry expressed in Lemma 1 the same construction applies to the right-hand side of the $y$-axis. Thus the $\operatorname{arc} b_{2}$ is followed by an arc $b_{3}$ with center at $(-1 / 2,0)$ and radius $r_{2}=g\left(r_{1}\right)$. By induction the whole solution is composed of a sequence of $\operatorname{arcs}\left(b_{i}\right)$ with radii $r_{i}$ satisfying (35). The relations (35) and (36) imply that the orbit converges to the periodic orbit described in the proof of Theorem 2.

Q.E.D. 


\section{FURTHER SOLUTION PROPERTIES FROM COMPUTER CONSTRUCTIONS}

Bounded periodic solutions of system (13), (14) exist for $\pi<\tau \leqslant 2 \pi$ (Theorems 2 and 3). Here we use computer-aided graphical constructions (according to the method described in Sect.4) to extend the analytical results on the behavior of solutions.

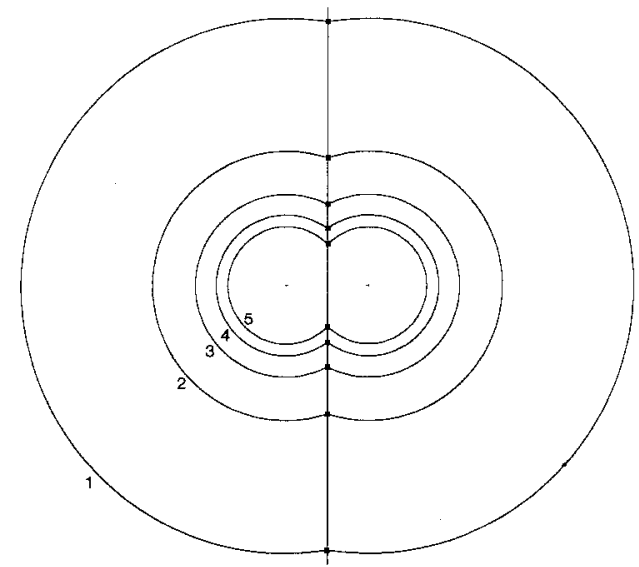

(a)

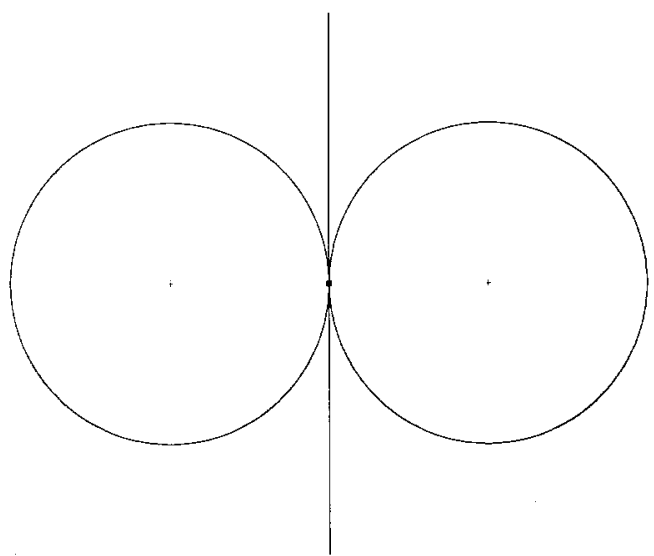

(b)

Fig. 4. (a) Five periodic orbits of Type $l$ associated with parameters $\Theta=0$ (always), $\tau=1.1 \pi(1), \tau=1.2 \pi(2), \tau=1.3 \pi(3), \tau=1.4 \pi(4), \tau=1.5 \pi$ (5). They can be obtained by choosing the initial condition $x_{0} \equiv 1, y_{0}=0$. Period of Type 1 solutions is $2 \tau$. (b) Here $\Theta=0$, $\tau=2 \pi$. Periodic orbit composed of two full circles. Initial condition $x_{0} \equiv 0, y_{0}=0$. According to numerical simulations this orbit appears to be unstable. 


\subsection{Varying $\tau$ with $\Theta=0$}

Three qualitatively different types of periodic solutions were found to occur for $\pi<\tau \leqslant 2 \pi$. These are illustrated in Figs. 4, 5, and 6 and are referred to, respectively, as Type 1 , Type 2 , and Type 3 . Type 1 solutions have their long axis along the $x$-axis and were described in the proof of Theorem 2. Type 3 solutions have their long axis oriented along the $y$-axis.

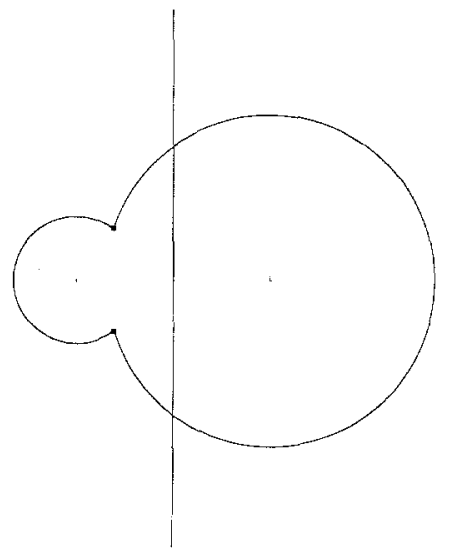

(a)

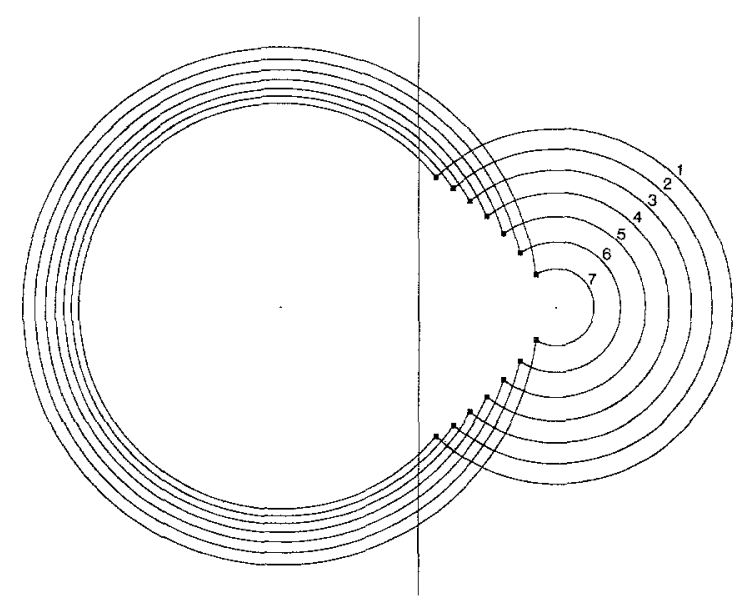

(b)

Fig. 5. (a) $\Theta=0, \tau=1.6 \pi$. Periodic orbit called left Type 2 in the text. Initial condition was $x_{0} \equiv 0.01, y_{0}=0$ (only the asymptotic behavior is shown). (b) Seven orbits of right Type 2 occuring for parameters $\tau=1.52 \pi(1), \tau=1.54 \pi(2), \tau=1.56 \pi(3), \tau=1.58 \pi(4), \tau=1.6 \pi(5)$, $\tau=1.62 \pi(6), \tau=1.64 \pi(7) ; \Theta=0$ throughout. Period of Type 2 solutions is $2 \tau$. At $\tau=3 \pi / 2$ they bifurcate from Type 1 solutions. 
There are two types of Type 2 solutions which are asymmetric with respect to the $y$-axis. The left Type 2 solutions have a small arc to the left and a large arc to the right (Fig. 5a), and vice versa for the right Type 2 solution (Fig. 5b).

Theorem 3 and all our computer-aided constructions indicate that Type 1 solutions are asymptotically orbitally stable if $\tau$ is not too large, i.e., when

$$
\pi<\tau<\tau_{\mathrm{c}}
$$

with some critical delay, $\tau_{\mathrm{c}}=1.5 \pi$. As $\tau$ increases along this range the diameter of the Type 1 solution in the phase plane projection decreases (Fig. 4a).

According to the simulations, at $\tau_{\mathrm{c}}$ a bifurcation takes place. Although Type 1 solutions continue to exist, for $\tau>\tau_{c}$ they are orbitally unstable (the extreme case when $\tau=2 \pi$ is shown in Fig. $4 \mathrm{~b}$ ). At $\tau=\tau_{\mathrm{c}}$ left and right Type 2 solutions bifurcate from the Type 1 solutions. Left and right Type 2 solutions appear to be locally asymptotically stable and their domains of attraction are separated by unstable Type 1 solutions. (Note added in proof: These numerical results have been proved in a forthcoming paper by an der Heiden and Reichard.)

Figure 5b shows several right Type 2 solutions for $\tau$ chosen between $1.52 \pi$ and $1.64 \pi$. With increasing $\tau$ the two points where the small and the large arc are connected move away from the $y$-axis (where they start with $\tau=\tau_{\mathrm{c}}$ ). As $\tau$ increases the diameter of the large arcs increases, and that of the small arc decreases. It can be shown analytically that these asymptotic solutions exist for values of $\tau$ between $3 \pi / 2$ and $5 \pi / 3$. The smaller arc has angular length $3 \pi-\tau$ and its radius is $\sin \left(\frac{1}{2}(5 \pi-3 \tau)\right) / \sin (\tau-\pi)$. The radius of the large arc is $\left(2 \cos \left(\frac{1}{2}(\tau-\pi)\right)^{-1}\right.$. The period of right Type 2 solutions is $2 \tau$. The same considerations hold for left Type 2 solutions.

The computer results suggest that for values $5 \pi / 3<\tau<2 \pi$ the majority of solutions is attracted by Type 3 periodic orbits. Type 3 orbits are composed of two arcs, each of which has angular length $\tau / 2$ (compare Fig. 6). Thus, the period is just $\tau$. Increasing $\tau$ increases the diameter of Type 3 solutions (Fig. 6), and in particular, as $\tau \rightarrow 2 \pi$ the diameter tends toward infinity.

Surprisingly Type 3 solutions exist also for all values of $\tau$ between $3 \pi / 2$ and $5 \pi / 3$, and the computer graphic results suggest that they are asymptotically orbitally stable for this range of $\tau$. Thus for each of these values of the parameter $\tau$ we have coexistence of at least four periodic orbits, three of which are asymptotically orbitally stable (left and right Type 2 and Type 3 ) and one (Type 1) is orbitally unbstable.

At the boundary between the domains of attraction of Type 2 and 
Type 3 solutions, very complicated behaviors exist. For example, Figs. 7a and $b$ show solutions corresponding to two (constant) initial conditions which differ only in the seventh decimal place and which are chosen close to this boundary region. In one case the solution converges toward a Type 2 orbit, whereas in the other it converges toward a Type 3 orbit.

For $\tau$ values in the interval $[2 \pi, 3 \pi]$ infinitely expanding spirals occur just as in the interval $(0, \pi]$, and no stable bounded solutions seem to exist. Our graphical results support the conjecture that this behavior holds for all values of $\tau$ satisfying

$$
2 n \pi<\tau<(2 n+1) \pi, \quad n \in \mathbb{N} \cup\{0\}
$$

On the other hand, if

$$
(2 n+1) \pi<\tau<(2 n+2) \pi, \quad n \in \mathbb{N} \cup\{0\}
$$

then asymptotically stable Type 1 and Type 3 solutions occur.

However, we could not find a Type 2 solution for values of $\tau>2 \pi$. Interestingly, we found many solutions which exhibited rather long and complicated types of transitory behaviours (a simple case is shown in Fig. 8a). These orbits, which could easily be mistaken as complicated periodic or aperiodic solutions, ultimately converged toward Type 3 attractors.

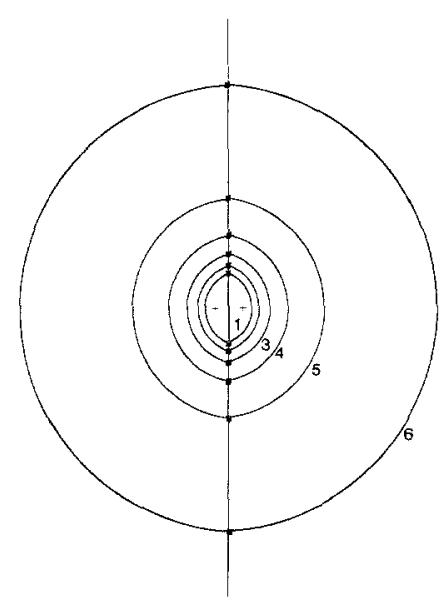

Fig. 6. Several Type 3 limit cycles. Parameters are $\Theta=0$ (always), $\tau=1.52 \pi(1), \tau=1.6 \pi(2)$, $\tau=1.68(3), \tau=1.76 \pi(4), \tau=1.94 \pi(5), \tau=1.92 \pi$ (6). Diameter of Type 3 orbits increases towards $\infty$ as $\tau \rightarrow 2 \pi$. Type 3 solutions have period $\tau$. 


\subsection{Varying $\Theta$}

Here we consider only the case when $\tau=3 \pi / 2$.

When $\Theta>1 / 2$ it is a trivial consequence of (15) that there are infinitely many periodic solutions, whose orbits in the $(x, y)$-plane are given by all circles with center at $(1 / 2,0)$ and radius smaller than $\Theta-1 / 2$.

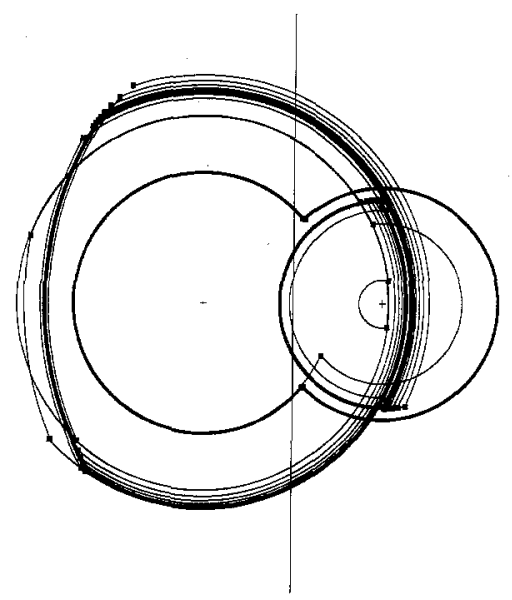

(a)

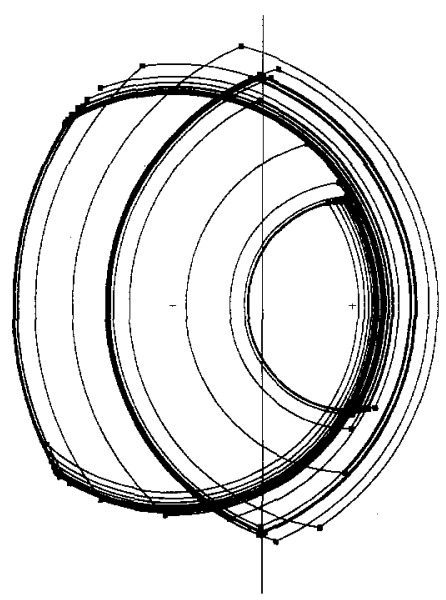

(b)

Fig. 7. (a) Transitory trajectory for $\Theta=0, \tau=1.52 \pi$. Initial condition $x_{0} \equiv 1, y_{0}=2.9628916$. Trajectory converges towards a periodic orbit of right Type 2. (b) Same parameters as in (a), but initial conditions $x_{0} \equiv 1, y_{0}=2.96289165$. Trajectory converges towards a periodic orbit of Type 3. 
These circles define a bounded center structure. Computer-aided constructions indicate that these bounded centers are (local) attractors. The limit orbit is generally not the largest orbit of the center.

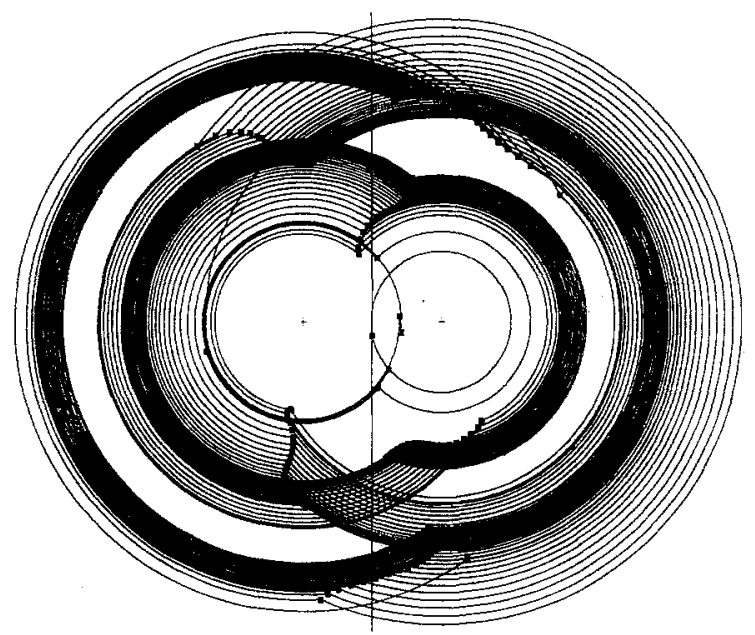

(a)

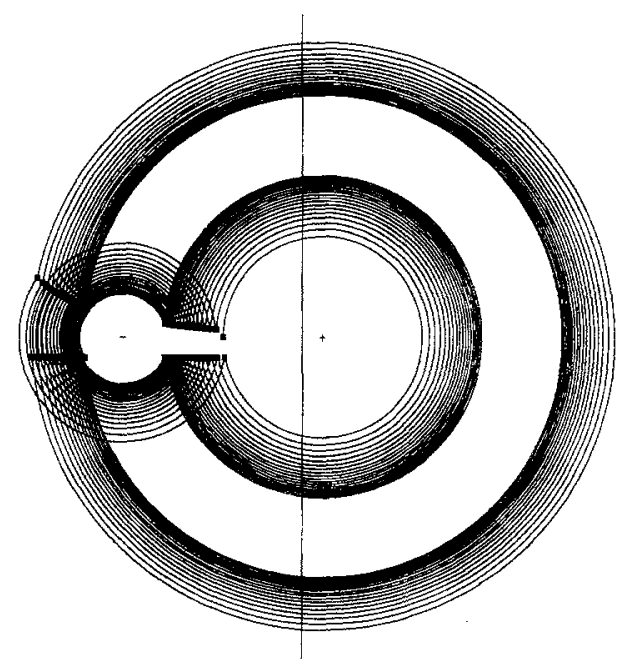

(b)

Fig. 8. (a) Transitory trajectory of the system described by Eqs. (13) and (14) with $\Theta=0$, $\tau=3.7 \pi$. Initial condition $x_{0} \equiv 0, y_{0}=0.1$. Trajectory is not chaotic, but converges, as $t \rightarrow \infty$, towards a periodic orbit of Type 3 as suggested by computer simulations. (b) Transitory trajectory in case of $\Theta=0.4, \tau=3 \pi / 2$. Initial condition $x_{0} \equiv 0, y_{0}=0$. 
A rather special situation arises for $\Theta=1 / 2$ where the threshold coincides with the upper level of the step function $f$. In this case, partially discussed earlier, complete insight is possible without use of a computer. Infinitely many different periodic orbits exist having a form similar to Fig. 1c. Each periodic orbit consists of three full circles, one large one and two smaller ones. If the small circle around $(1 / 2,0)$ has radius $r$, then the small circle around $(-1 / 2,0)$ has radius $1-r$. The large circle whose center is $(1 / 2,0)$ has the radius $2-r$. The values of $r$ range from 0 to 1 . Figure 1c shows the case $r=1 / 4$. In the limiting situation with $r=1$ the second small circle degenerates to a point $(=(-1 / 2,0))$, and the two circles around $(1 / 2,0)$ coincide. Thus only a single circle (with radius 1 ) remains. Note, however, that within one single period, which for all $r$ is $6 \pi=4 \tau$, the solution is constant in two time intervals, each of length $\pi$. During this time the solution $(x(t), y(t))$ stays at the point $(-1 / 2,0)$, which represents the degenerate circle.

For $\Theta$ values smaller than $1 / 2$ and larger than 0 , again (locally asymptotically orbitally stable) left and right Type 2 solutions exist. A series of $\Theta$ values would yield a picture like Fig. 5b. Since it is difficult to imagine how these Type 2 solutions bifurcate from the peculiar solutions at $\Theta=1 / 2$, we illustrate a transient solution for $\Theta=0.4$ in Fig. 8b. At $\Theta=0$ left and right Type 2 solutions melt into a single Type 1 solution.

For negative values of $\Theta$ the situation is analogous to that of the corresponding positive value.

\section{DISCUSSION}

Here we have studied the special case of a nonlinear second-order delay-differential equation with no damping $\left(a_{1}=0\right)$ and piecewise constant negative feedback. This equation is of interest because it is one of the very few nonlinear second-order delay-differential equations which can be investigated analytically. Moreover, solutions can be constructed in a simple geometric fashion. We have shown that this equation can produce a variety of stable periodic limit cycle solutions which have one maxima per period (i.e., simple type).

An interesting feature of the solutions of (12) (or 14) is the existence of long transients for certain parameter ranges which can last for 100's of delay times (Figs. 7a and $b, 8$ ). We believe that the reason for this is the coexistence of several locally stable limit cycles for certain parameter configurations $(\tau, \Theta)$. Since the state space of delay differential equations, consisting of initial functions on the interval $[-\tau, 0]$, has infinite dimension, an arbitrary initial function has a high probability to be far away from all of the several stable limit cycles. In particular, if the initial condi- 
tion is sufficiently close to, but not on, the boundary between the attraction basins of two stable limit cycles, then the transitory behavior can have an arbitrarily long duration. Of course, it would be highly desirable to have more detailed insight into the origin and structure of these transitory solutions than is presented here. Preliminary computer simulations of (5) with positive damping $\left(a_{1}>0\right)$ and piecewise constant negative feedback indicate that long transients also occur (data not shown). Furthermore, in the case of negative damping $\left(a_{1}<0\right)$ the transients can be extremely long. It is in fact quite surprising that despite this added dynamical complexity, the solutions in (5) are attracted to simple limit cycles. Such long transients have not been observed in system (3) with piecewise constant negative feedback.

Although in this paper we have emphasized the importance of secondorder nonlinear delay-differential equations for a number of applications, it must be realized that the analysis of third- and even higher-order delay equations is also likely to be of importance. For example, for the pupil light reflex, measurements of the open loop transfer function indicate that a third-order nonlinear delay-differential equation is likely to be most relevant for understanding the properties of this neural reflex arc (Stark, 1959). Thus we are led back to Eq. (1). There appear to be a wealth of interesting problems requiring the attention of mathematicians interested in the analysis of nonlinear delay-differential equations.

\section{ACKNOWLEDGMENT}

We thank Dr. Klaus Reichard, University of Witten/Herdecke, for interesting discussions in the context of this paper and for help in the production of the figures.

\section{REFERENCES}

Bejczy, A. K., and Salisbury, J. K., Jr. (1983). Controlling remote manipulations through kinesthetic coupling. Comp. Mech. Eng. July, 48-60.

Beuter, A., Larocque, D., and Glass, L. Complex oscillations in a human motor system. J. Motor Behav. (submitted for publication).

Blythe, S. P., Nisbet, R. M., and Gurney, W. S. C. (1982). Instability and complex dynamic behaviour in population models. Theor. Pop. Biol. 22, 147-176.

Chow, S.-N. (1974). Existence of periodic solutions of autonomous functional differential equations. J. Diff. Eq. 15, 350-378.

Coleman, B. D., and Renninger, G. H. (1974). Theory of delayed lateral inhibition in the compound eye of Limulus. Proc. Natl. Acad. Sci. USA 71, 2887-2891.

Furumochi, T. (1978). Existence of periodic solutions of one-dimensional differential-delay equations. Tohoku Math. J. 30, 13-35.

Gibbs, H. M., Hopf, F. A., Kaplan, D. L., and Shoemaker, R. L. (1981). Observation of chaos in optical bistability. Phys. Rev. Lett. 46, 474-477. 
Glass, L., and Mackey, M. C. (1979). Pathological conditions resulting from instabilities in physiological control systems. Ann. N.Y. Acad. Sci. 316, 214-235.

Glass, L., Beuter, A., and Larocque, D. (1988). Time delays, oscillations and chaos in physiological control systems. Math. Biosci. 90, 111-115.

Gurney, W. S. C., Blythe, S. P., and Nisbet, R. M. (1980). Nicholson's blowflies revisited. Nature 287, 17-21.

Hadeler, K. P. (1980). Effective computation of periodic orbits and bifurcation diagrams in delay equations. Numer. Math. 34, 457-467.

Hadeler, K. P., and Tomiuk, J. (1977). Periodic solutions of difference-differential equations. Arch. Rat. Mech. Anal. 65, 87-95.

an der Heiden, U. (1979a). Delays in physiological systems. J. Math. Biol. 8, 345-364.

an der Heiden, U. (1979b). Periodic solutions of a nonlinear second order differential equation with delay. J. Math. Anal. Appl. 70, 599-609.

an der Heiden, U. (1985). Stochastic properties of simple differential-delay equations. In Meinardus, G., and Nürnberger, G. (eds.), Delay Equations, Approximation and Application, Birkhäuser Verlag, Basel-Boston-Stuttgart.

an der Heiden, U., and Mackey, M. C. (1982). The dynamics of production and destruction: Analytic insight into complex behaviour. J. Math. Biol. 16, 75-101.

an der Heiden, U., and Reichard, K. Bifurcation and stability of oscillations in certain nonlinear second order differential equations with retarded argument (in preparation).

an der Heiden, U., and Walther, H.-O. (1983). Existence of chaos in control systems with delayed feedback. J. Diff. Eq. 47, 273-295.

Hutchinson, G. E. (1948). Circular causal systems in ecology. Ann. N.Y. Acad. Sci. 50, 221.

Ikeda, K., Daido, H., and Akimoto, O. (1980). Optical turbulence: Chaotic behaviour of transmitted light from a ring cavity. Phys. Rev. Lett. 45, 709-712.

Kaplan, J. L., and Yorke, J. A. (1977). On the nonlinear differential delay equation $x^{\prime}(t)=$ $-f(x(t), x(t-1))$. J. Diff. Eq. 23, 293-314.

Li, T. Y., and Yorke, J. A. (1975). Period three implies chaos. Am. Math. Month. 82, 985-992.

Longtin, A., and Milton, J. G. (1988). Complex oscillations in the human pupil light reflex with "mixed" and delayed feedback. Math. Biosci. 90, 183-199.

Longtin, A., and Milton, J. G. (1989a). Insight into the transfer function, gain and oscillation onset for the pupil light reflex using nonlinear delay-differential equations. Biol. Cybern. 61, 51-58.

Longtin, A., and Milton, J. G. (1989b). Modelling autonomous oscillations in the human pupil light reflex using nonlinear delay-differential equations. Bull. Math. Biol. 51, 605.

Mackey, M. C' (1978). A unified hypothesis for the origin of aplastic anemia and periodic hematopoiesis. Blood 51, 941-956.

Mackey, M. C. (1979). Periodic auto-immune hemolytic anemia: An induced dynamical disease. Bull. Math. Biol. 41, 829-834.

Mackey, M. C. (1990). Commodity price fluctuations: Price dependent delays and nonlinearities as explanatory factors. $J$. Econ. Theory $48,497$.

Mackey, M. C., and an der Heiden, U. (1982). Dynamical diseases and bifurcations: Understanding functional disorders in physiological systems. Funkt. Biol. Med. 1, 156-164.

Mackey, M. C., and an der Heiden, U. (1984). The dynamics of recurrent inhibition. J. Math. Biol. 19, 211-225.

Mackey, M. C., and Glass, L. (1977). Oscillation and chaos in physiological control systems. Science 197, 87-89.

Mackey, M. C., and Milton, J. G. (1987). Dynamical diseases. Ann. N.Y. Acad. Sci. 504, 16-32. 
Marcus, C. M., and Westervelt, R. M. (1989). Stability of analog neural networks with delay. Phys. Rev. A 39, 347-359.

May, R. (1976). Simple mathematical models with very complicated dynamics. Nature 261, $459-467$.

May, R. (1980). Mathematical models in whaling and fisheries management. Lect. Math. Life Sci. 13, 1-64.

Maynard Smith, J. (1968). Mathematical Ideas in Biology, Cambridge University Press, Cambridge, pp. 98-104.

Metropolis, N., Stein, M. L., and Stein, P. R. (1973). On finite limit sets for transformations on the unit interval. J. Combinator. Theory (A) 15, 25-44.

Milton, J. G., and Longtin, A. (1989). Clamping the pupil light reflex with external feedback: Evaluation of constriction and dilation from cycling measurements (sumitted for publication).

Milton, J. G., Longtin, A., Kirkham, T. H., and Francis, G. S. (1988). Irregular pupil cycling as a characteristic abnormality in patients with demyelinative optic neuropathy. $A m . J$. Ophthalmol. 105, 402-407.

Milton, J. G., Longtin, A., Beuter, A., Mackey, M. C., and Glass, L. (1989). Complex dynamics and bifurcations in neurology. J. Theoret. Biol. 138, 129.

Minorsky, N. (1962). Nonlinear Oscillations, Van Nostrand, Princeton, N.J., pp. 534-536.

Misiurewicz, M., and Szlenk, W. (1980). Entropy of piecewise monotone mappings. Studia Math. 67, 45-63.

Nussbaum, R. D. (1974). Periodic solutions of some nonlinear autonomous functional differential equations. Ann. Mat. pura appl. 101, 263-306.

Peters, H. (1980). Comportment chaotique d'une équation' differentielle retardée. C.R. Acad. Sci. Paris 290, 1119-1122.

Phillips, C. A. (1988). Sensory feedback control of upper- and lower-extremity motor prostheses. CRC Crit. Rev. Biomed. Eng. 16, 105-140.

Reichardt, W., and Poggio, T. (1976). Visual control of orientation behavior of the fly. Q. Rev. Biophys. 9, 311-438.

Sarkovskii, A. N. (1964). Coexistence of cycles of a continuous map of a line into itself. $U k$ ran. Math. J. 16, 61-71.

Saupe, D. (1982). Beschleunigte PL-Kontinuitätsmethoden und periodische Lösungen parametrisierter Differentialgleichungen mit Zeitverzögerung, Dissertation, Universität Bremen, Bremen.

Stark, L. (1959). Stability, oscillations and noise in the human pupil servomechanism. Proc. IRE 47, 1925-1939.

Taylor, C. E., and Sokal, R. R. (1976). Oscillations in housefly population sizes due to time lags. Ecology 57, 1060-1067.

Vallée, R., Dubois, P., Côté, M., and Delisle, C. (1987). Second-order differential-delay equation to describe a hybrid bistable device. Phys. Rev. A 36, 1327-1332.

Walther, H.-O. (1981a). Density of slowly oscillating solutions of $\dot{x}(t)=-f(x(t-1)) . J$. Math. Anal. Appl. 79, 127-140.

Walther, H.-O. (1981b). Homoclinic solution and chaos in $\dot{x}(t)=f(x(t-1))$. J. Nonlin. Anal. $5,775-788$.

Wazewska-Czysewska, M., and Lasota, A. (1976). Matematyczne problemy dynamiki ukladu krwinek czerwonych. Mat. Stosowana 6, 23-40. 